PROCEEDINGS OF THE

AMERICAN MATHEMATICAL SOCIETY

Volume 126, Number 7, July 1998, Pages 1949-1953

S 0002-9939(98)04422-0

\title{
p-INTEGRAL BASES OF A CUBIC FIELD
}

\author{
ŞABAN ALACA
}

(Communicated by William W. Adams)

\begin{abstract}
A $p$-integral basis of a cubic field $K$ is determined for each rational prime $p$, and then an integral basis of $K$ and its discriminant $d(K)$ are obtained from its $p$-integral bases.
\end{abstract}

\section{INTRODUCTION}

Let $K=Q(\theta)$ be an algebraic number field of degree $n$, and let $O_{K}$ denote the ring of integral elements of $K$. If $O_{K}=\alpha_{1} Z+\alpha_{2} Z+\cdots+\alpha_{n} Z$, then $\left\{\alpha_{1}, \alpha_{2}, \ldots, \alpha_{n}\right\}$ is said to be an integral basis of $K$. For each prime ideal $P$ and each nonzero ideal $A$ of $K, \nu_{P}(A)$ denotes the exponent of $P$ in the prime ideal decomposition of $A$.

Let $P$ be a prime ideal of $K$, let $p$ be a rational prime, and let $\alpha \in K$. If $\nu_{P}(\alpha) \geq 0$, then $\alpha$ is called a $P$-integral element of $K$. If $\alpha$ is $P$-integral for each prime ideal $P$ of $K$ such that $P \mid p O_{K}$, then $\alpha$ is called a $p$-integral element of $K$. Let $\left\{\omega_{1}, \omega_{2}, \ldots, \omega_{n}\right\}$ be a basis of $K$ over $Q$, where each $\omega_{i}(1 \leq i \leq$ $n$ ) is a $p$-integral element of $K$. If every $p$-integral element $\alpha$ of $K$ is given as $\alpha=a_{1} \omega_{1}+a_{2} \omega_{2}+\cdots+a_{n} \omega_{n}$, where the $a_{i}$ are $p$-integral elements of $Q$, then $\left\{\omega_{1}, \omega_{2}, \ldots, \omega_{n}\right\}$ is called a $p$-integral basis of $K$.

In Theorem 2.1 a $p$-integral basis of a cubic field $K$ is determined for every rational prime $p$, and in Theorem 2.2 an integral basis of $K$ is obtained from its $p$-integral bases.

Let $K=Q(\theta)$, where $\theta$ is a root of the irreducible polynomial

$$
x^{3}-a x+b=0, \quad a, b \in Z \text { with } \nu_{p}(a)<2 \text { or } \nu_{p}(b)<3
$$

see [2, p. 579]. The discriminant of $\theta$ is $\Delta=4 a^{3}-27 b^{2}$ and $\Delta=i(\theta)^{2} d(K)$, where $d(K)$ denotes the discriminant of $K$, and $i(\theta)$ is the index of $\theta$. For each rational prime $p$, set $s_{p}=\nu_{p}(\Delta)$ and $\Delta_{p}=\Delta / p^{s_{p}}$.

The following three theorems are the special cases for $n=3$ of Theorem 2.1, Theorem 3.1 and Theorem 3.3, respectively, given in [1].

Theorem 1.1. Let $K=Q(\theta)$ be a cubic field, where $\theta$ is a root of the irreducible polynomial (1.1). Let $p$ be a rational prime, and let $\alpha=\left(x+y \theta+\theta^{2}\right) / p^{m}$, where

Received by the editors December 26, 1996.

1991 Mathematics Subject Classification. Primary 11R16, 11R29.

Key words and phrases. Cubic field, p-integral basis, integral basis, discriminant.

(C)1998 American Mathematical Society 
$x, y, m \in Z, m \geq 0$. Set

$$
\begin{aligned}
& X=3 x+2 a, \\
& Y=3 x^{2}+4 a x-a y^{2}+3 b y+a^{2}, \\
& Z=x^{3}+2 a x^{2}-a x y^{2}+3 b x y+a^{2} x-b y^{3}+a b y+b^{2} .
\end{aligned}
$$

Then $\alpha$ is a p-integral if and only if

$$
X \equiv 0\left(\bmod p^{m}\right), \quad Y \equiv 0\left(\bmod p^{2 m}\right), \quad Z \equiv 0\left(\bmod p^{3 m}\right) .
$$

Theorem 1.2. Let $K=Q(\theta)$ be a cubic field, where $\theta$ is a root of the irreducible polynomial (1.1). Let $p$ be a rational prime, and let

$$
\frac{u+\theta}{p^{i}} \quad(u \in Z) \quad \text { and } \quad \frac{x+y \theta+\theta^{2}}{p^{j}} \quad(x, y \in Z)
$$

be $p$-integral in $K$ with the integers $i$ and $j$ as large as possible. Then

$$
\left\{1, \frac{u+\theta}{p^{i}}, \frac{x+y \theta+\theta^{2}}{p^{j}}\right\}
$$

is a p-integral basis of $K$, and

$$
\nu_{p}(d(K))=\nu_{p}(\Delta)-2(i+j) .
$$

Theorem 1.3. Let $K=Q(\theta)$ be a cubic field, where $\theta$ is a root of the irreducible polynomial (1.1). If there are no rational primes dividing $i(\theta)$, then $\left\{1, \theta, \theta^{2}\right\}$ is an integral basis of $K$. Let $p_{1}, p_{2}, \ldots, p_{s}$ be the distinct primes dividing $i(\theta)$. Let

$$
\left\{1, \frac{x_{r, 0}^{(1)}+\theta}{p_{r}^{k_{r, 1}}}, \frac{x_{r, 0}^{(2)}+x_{r, 1}^{(2)} \theta+\theta^{2}}{p_{r}^{k_{r, 2}}}\right\}
$$

be a $p_{r}$-integral basis of $K(r=1,2, \ldots, s)$ as given in Theorem 1.2. Define the integers $X_{i}^{(j)}(i=0,1, \ldots, j-1, j=1,2)$ by

$$
X_{i}^{(j)} \equiv x_{r, i}^{(j)}\left(\bmod p_{r}^{k_{r, j}}\right) \quad(r=1,2, \ldots, s),
$$

and let $T_{j}=\prod_{r=1}^{s} p_{r}^{k_{r, j}}(j=1,2)$. Then an integral basis of $K i s$

$$
\left\{1, \frac{X_{0}^{(1)}+\theta}{T_{1}}, \frac{X_{0}^{(2)}+X_{1}^{(2)} \theta+\theta^{2}}{T_{2}}\right\} \text {. }
$$

2. $p$-INTEGRAL BASES OF A CUBIC FIELD

Theorem 2.1. Let $K=Q(\theta)$ be a cubic field, where $\theta$ is a root of the irreducible polynomial (1.1). Then a 2-integral basis, a 3-integral basis, and a $p(>3)$-integral basis of $K$ are given in Table $\mathrm{A}$, Table $\mathrm{B}$, and Table $\mathrm{C}$, respectively. (Note that the notation $a \equiv b(\bmod m)$ has been shortened to $a \equiv b(m)$ in the tables.)

Proof. The ideas of the proof are illustrated in one case for each table.

A: $a \equiv 1(\bmod 4)$ and $b \equiv 2(\bmod 4)$. By Theorem $1.1,\left(x+y \theta+\theta^{2}\right) / 2$ is not a 2 -integral element of $K$ for any pair of integers $x, y$. Thus by Theorem $1.2,\left\{1, \theta, \theta^{2}\right\}$ is a 2-integral basis of $K$ and $\nu_{2}(d(K))=s_{2}=3$.

B: $a \equiv 3(\bmod 9), \nu_{3}(b)=0, b^{2} \equiv 4(\bmod 9), b^{2} \not \equiv a+1(\bmod 27)$. Then $s_{3}=$ $\nu_{3}(\Delta)=5$. By Theorem 1.1, $(x+\theta) / 3$ is not a 3-integral element of $K$ for any rational integer $x$, and $\left(1-b \theta+\theta^{2}\right) / 3$ is a 3 -integral element of $K$. One can also see that $\left(x+y \theta+\theta^{2}\right) / 3^{2}$ is not a 3 -integral element of $K$ for any pair of integers 
TABle A

\begin{tabular}{||l|l|c|c||}
\hline Condition & 2-integral basis & $s_{2}$ & $\nu_{2}(d(K))$ \\
\hline \hline$b \equiv 1(2)$ & $\left\{1, \theta, \theta^{2}\right\}$ & 0 & 0 \\
\hline$a \equiv 0(2), b \equiv 2(4)$ & $\left\{1, \theta, \theta^{2}\right\}$ & 2 & 2 \\
\hline$a \equiv 0(2), b \equiv 4(8)$ & $\left\{1, \theta, \theta^{2} / 2\right\}$ & 4 & 4 \\
\hline$a \equiv 2(4), b \equiv 0(8)$ & $\left\{1, \theta, \theta^{2} / 2\right\}$ & 5 & 3 \\
\hline$a \equiv 1(4), b \equiv 0(4)$ & $\left\{1, \theta,\left(\theta+\theta^{2}\right) / 2\right\}$ & 2 & 0 \\
\hline$a \equiv 3(4), b \equiv 0(4)$ & $\left\{1, \theta, \theta^{2}\right\}$ & 2 & 2 \\
\hline$a \equiv 1(4), b \equiv 2(4)$ & $\left\{1, \theta, \theta^{2}\right\}$ & 3 & 3 \\
\hline$a \equiv 3(4)$ & $\left\{1, \theta,\left(x+y \theta+\theta^{2}\right) / 2^{m}\right\}$ & $s_{2} \geq 5$ & 3 \\
$b \equiv 2(4)$ & $m=\left(s_{2}-3\right) / 2$ & $s_{2} \equiv 1(2)$ & \\
$s_{2} \equiv 1(2)$ & $3 x \equiv-2 a\left(2^{m}\right)$ & & \\
& $a y \equiv 3(b / 2)\left(2^{m}\right)$ & & \\
\hline$a \equiv 3(4)$ & $\left\{1, \theta,\left(x+y \theta+\theta^{2}\right) / 2^{m}\right\}$ & $s_{2} \geq 4$ & 2 \\
$b \equiv 2(4)$ & $m=\left(s_{2}-2\right) / 2$ & $s_{2} \equiv 0(2)$ & \\
$s_{2} \equiv 0(2)$ & $3 x \equiv-2 a\left(2^{m}\right)$ & & \\
$\Delta_{2} \equiv 3(4)$ & $a y \equiv 3(b / 2)\left(2^{m}\right)$ & & \\
\hline$a \equiv 3(4)$ & $\left\{1, \theta,\left(x+y \theta+\theta^{2}\right) / 2^{m+1}\right\}$ & $s_{2} \geq 4$ & 0 \\
$b \equiv 2(4)$ & $m=\left(s_{2}-2\right) / 2$ & $s_{2} \equiv 0(2)$ & \\
$s_{2} \equiv 0(2)$ & $3 x \equiv-2 a\left(2^{m+1}\right)$ & & \\
$\Delta_{2} \equiv 1(4)$ & $a y \equiv 3(b / 2)+2^{m}\left(2^{m+1}\right)$ & & \\
\hline
\end{tabular}

Table B

\begin{tabular}{||l|l|c|c||}
\hline Condition & 3-integral basis & $s_{3}$ & $\nu_{3}(d(K))$ \\
\hline \hline$\nu_{3}(a)=0$ & $\left\{1, \theta, \theta^{2}\right\}$ & 0 & 0 \\
\hline$\nu_{3}(a)=\nu_{3}(b)=1$ & $\left\{1, \theta, \theta^{2}\right\}$ & 3 & 3 \\
\hline $1=\nu_{3}(b)<\nu_{3}(a)$ & $\left\{1, \theta, \theta^{2}\right\}$ & 5 & 5 \\
\hline $2=\nu_{3}(b)=\nu_{3}(a)$ & $\left\{1, \theta, \theta^{2} / 3\right\}$ & 6 & 4 \\
\hline $2=\nu_{3}(b)<\nu_{3}(a)$ & $\left\{1, \theta, \theta^{2} / 3\right\}$ & 7 & 5 \\
\hline $1=\nu_{3}(a)<\nu_{3}(b)$ & $\left\{1, \theta, \theta^{2} / 3\right\}$ & 3 & 1 \\
\hline$\nu_{3}(b)=0, \nu_{3}(a) \geq 1$ & $\left\{1, \theta,\left(1-b \theta+\theta^{2}\right) / 3\right\}$ & 3 & 1 \\
$a \neq 3(9)$ & & & \\
$b^{2} \equiv a+1(9)$ & & 3 & 3 \\
\hline$\nu_{3}(b)=0, \nu_{3}(a) \geq 1$ & $\left\{1, \theta, \theta^{2}\right\}$ & & \\
$a \neq \equiv(9)$ & & 5 & 3 \\
$b^{2} \not \equiv a+1(9)$ & $\left\{1, \theta,\left(1-b \theta+\theta^{2}\right) / 3\right\}$ & & \\
\hline$\nu_{3}(b)=0$ & & & \\
$a \equiv 3(9)$ & & 4 & \\
$b^{2} \equiv 4(9)$ & & & \\
$b^{2} \not \equiv a+1(27)$ & $\left\{1, \theta, \theta^{2}\right\}$ & & \\
\hline$\nu_{3}(b)=0$ & & & \\
$a \equiv 3(9)$ & & & \\
$b^{2} \not \equiv 4(9)$ & $\{1,(b+\theta) / 3$, & & \\
\hline$\nu_{3}(b)=0$ & $\left.\left(x+y \theta+\theta^{2}\right) / 3^{m}\right\}$ & & \\
$a \equiv 3(9)$ & $m=[(s 3-2) / 2]$ & & \\
$b^{2} \equiv a+1(27)$ & $2 a y \equiv 3 b\left(3_{3} / 2\right]$ \\
& & & \\
& & & \\
\hline
\end{tabular}




\section{TABLE C}

\begin{tabular}{||l|l|c|c||}
\hline Condition & $p(>3)$-integral basis & $s_{p}$ & $\nu_{p}(d(K))$ \\
\hline \hline$\nu_{p}(a)=0, \nu_{p}(b) \geq 1$ & $\left\{1, \theta, \theta^{2}\right\}$ & 0 & 0 \\
or & & & \\
$\nu_{p}(a) \geq 1, \nu_{p}(b)=0$ & & & \\
\hline $1=\nu_{p}(b) \leq \nu_{p}(a)$ & $\left\{1, \theta, \theta^{2}\right\}$ & 2 & 2 \\
\hline $2=\nu_{p}(b) \leq \nu_{p}(a)$ & $\left\{1, \theta, \theta^{2} / p\right\}$ & 4 & 2 \\
\hline $1=\nu_{p}(a)<\nu_{p}(b)$ & $\left\{1, \theta, \theta^{2} / p\right\}$ & 3 & 1 \\
\hline$\nu_{p}(a)=\nu_{p}(b)=0$ & $\left\{1, \theta,\left(x+y \theta+\theta^{2}\right) / p^{m}\right\}$ & $s_{p} \geq 0$ & $s_{p}-2\left[s_{p} / 2\right]$ \\
& $m=\left[s_{p} / 2\right]$, \\
& $3 x \equiv-2 a\left(p^{m}\right)$, & & \\
& $2 a y \equiv 3 b\left(p^{m}\right)$ & & \\
\hline
\end{tabular}

$x, y$. Thus, by Theorem 1.2, $\left\{1, \theta,\left(1-b \theta+\theta^{2}\right) / 3\right\}$ is a 3 -integral basis of $K$ and $\nu_{3}(d(K))=3$.

C: $\nu_{p}(a)=\nu_{p}(b)=0$. Set $U=9 b y-2 a^{2}$ and $V=2 a y-3 b$. Then $Y=$ $\left(2^{2} a^{2} X^{2}-U^{2}-3 \Delta y^{2}\right) / 2^{2} 3 a^{2}$ and $Z=\left(2^{2} a^{3} X^{3}-3 a X U^{2}-3^{2} a \Delta X y^{2}-3 U^{3}-\right.$ $\left.3^{2} \Delta U y^{2}+2 a \Delta U+3^{3} b V^{3}+23^{2} b \Delta V-\Delta^{2}\right) / 2^{2} 3^{3} a^{3}$.

Let $m=\left[s_{p} / 2\right]$. Define integers $x$ and $y$ by $3 x \equiv-2 a\left(\bmod p^{m}\right)$ and $2 a y \equiv$ $3 b\left(\bmod p^{m}\right)$, respectively. Note that $p^{2 m} \mid \Delta$. Then

$$
3 b U=\left(4 a^{3}-\Delta\right) y-6 a^{2} b \equiv 0\left(\bmod p^{m}\right) .
$$

So, $U \equiv 0\left(\bmod p^{m}\right)$. Hence, $X \equiv 0\left(\bmod p^{m}\right), Y \equiv 0\left(\bmod p^{2 m}\right)$, and $Z \equiv 0\left(\bmod p^{3 m}\right)$. Thus, by Theorem 1.1, $\left(x+y \theta+\theta^{2}\right) / p^{m}$ is a $p$-integral element of $K$. Therefore, by Theorem 1.2, $\left\{1, \theta,\left(x+y \theta+\theta^{2}\right) / p^{m}\right\}$ is a $p$-integral basis of $K$, and $\nu_{p}(d(K))=$ $s_{p}-2\left[s_{p} / 2\right]$.

Remark 2.1. Note that for any rational prime $p$, a $p$-integral basis of $K$ is given in the form $\left\{1, \theta,\left(R_{p}+S_{p} \theta+\theta^{2}\right) / p^{T_{p}}\right\}$ except in the case

$$
\nu_{3}(b)=0, \quad a \equiv 3(\bmod 9), \quad b^{2} \equiv a+1(\bmod 27)
$$

when a 3 -integral basis is of the form $\left\{1,(b+\theta) / 3,\left(R_{3}+S_{3} \theta+\theta^{2}\right) / 3^{T_{3}}\right\}$. Furthermore, for only finitely many rational primes $p, T_{p}$ is nonzero.

Remark 2.2. The discriminant of a cubic field given in [2, Theorem 2] follows from Theorem 2.1.

The following theorem follows from Theorem 1.3 and Theorem 2.1.

Theorem 2.2. Let $K=Q(\theta)$ be a cubic field, where $\theta$ is a root of the irreducible polynomial (1.1). For every rational prime $p$, set $R_{p}, S_{p}$ and $T_{p}$ as in Remark 2.1. Let $R$ and $S$ be integers such that for all primes $p$

$$
R \equiv R_{p}\left(\bmod p^{T_{p}}\right) \quad \text { and } S \equiv S_{p}\left(\bmod p^{T_{p}}\right) .
$$

Let $T$ be the positive integer $T=\prod_{p} p^{T_{p}}$. Then

$$
\left\{1, \theta, \frac{R+S \theta+\theta^{2}}{T}\right\}
$$

is an integral basis of $K$ except in the case

$$
\nu_{3}(b)=0, \quad a \equiv 3(\bmod 9), \quad b^{2} \equiv a+1(\bmod 27)
$$


when an integral basis is

$$
\left\{1, \frac{b+\theta}{3}, \frac{R+S \theta+\theta^{2}}{T}\right\} .
$$

\section{ExAmples}

Example 3.1. Let $K=Q(\theta)$, where $\theta^{3}-\theta+4=0$. Here $a=1$ and $b=4$. Then $\Delta=-2^{2} \cdot 107$. Hence $s_{2}=2, s_{107}=1$ and $s_{p}=0$ for every rational prime $p \neq 2,107$. So $R_{2}=0, S_{2}=1$ and $T_{2}=1$. Therefore $R=0, S=1$ and $T=2$. Hence $\left\{1, \theta,\left(\theta+\theta^{2}\right) / 2\right\}$ is an integral basis of $K$ and $d(K)=-107$.

Example 3.2. Let $K=Q(\theta)$, where $\theta^{3}-255 \theta+3850=0$. Here $a=255$ and $b=3850$. Then $\Delta=-2^{4} \cdot 3^{6} \cdot 5^{3} \cdot 229$. So, $R_{2}=2, S_{2}=-1, T_{2}=1, R_{3}=$ $1, S_{3}=2, T_{3}=2, R_{5}=0, S_{5}=0$, and $T_{5}=1$. Therefore $R=10, S=-25$, and $T=90$. Hence $\left\{1,(3850+\theta) / 3,\left(10-25 \theta+\theta^{2}\right) / 90\right\}$ is an integral basis of $K$, and $d(K)=-2^{2} \cdot 5 \cdot 229$.

Example 3.3. Let $K=Q(\beta)$, where $\beta^{3}-\beta^{2}-82 \beta+311=0$. Set $\theta=3 \beta-1$. Then $K=Q(\beta)=Q(\theta)$, and $\theta^{3}-741 \theta+7657=0$. Hence, $\Delta=3^{6} \cdot 13^{2} \cdot 19^{2}$. Then $R_{3}=1, S_{3}=2$, and $T_{3}=2$. Therefore, $R, S$, and $T$ can be taken as $R=1, S=1$ and $T=3^{2}$. Hence

$$
\left\{1,(1+\theta) / 3,\left(1+2 \theta+\theta^{2}\right) / 3^{2}\right\}=\left\{1, \beta, \beta^{2}\right\}
$$

is an integral basis of $K$, and $d(K)=13^{2} \cdot 19^{2}$.

\section{REFERENCES}

1. S.. Alaca, p-Integral Bases of Algebraic Number Fields, submitted for publication.

2. P. Llorente and E. Nart, Effective determination of the decomposition of the rational primes in a cubic field, Proc. Amer. Math. Soc. 87 (1983), 579-585. MR 84d:12003

Centre for Research in Algebra and Number Theory, Department of Mathematics and Statistics, Carleton University, Ottawa, Ontario, Canada K1S 5B6

E-mail address: salaca@math.carleton.ca 